Medizinprodukte in der ästhetischen Dermatologie

\title{
Allergan startet Qualitätsinitiative
}

\section{Auch bei Medizinprodukten spielt die Qualität eine ausschlaggebende Rolle - dies zeigte jüngst der Skan- dal um Brustimplantate. Das Unter- nehmen Allergan hat daher eine Qualitätsinitiative gestartet, um Ärzte und Patienten für das Thema Qualität bei Medizinprodukten zu sensibilisieren.}

Immer noch steigt die Nachfrage nach ästhetischen Eingriffen stetig an. Wie Dr. Mauricio de Maio, plastischer Chirurg an der „Faculty of Medicine of the University of Sao Paulo, Brasilien, anführte, gab der Skandal um die PIPBrustimplantate den Ausschlag für die Qualitätsinitiative des Unternehmens, die in acht europäischen Ländern gestartet wird. Künftig sollen Patienten eine aktivere Rolle spielen, wenn es um die Auswahl der eingesetzten Medizinprodukte geht. So sollen sie angeregt werden, gezielt nach dem verwendeten Produkt zu fragen, sowie nach wissen- schaftlichen Erkenntnisse, die für dessen Einsatz den Ausschlag gaben. Weitere Fragen zielen auf den Hersteller, die Häufigkeit der Verwendung und auf das gewünschte Behandlungsergebnis. Diese fünf Fragen sollen künftig Bestandteil des Arztgesprächs sein.

\section{Aufklärungsgespräche sind wichtig}

Das Arztgespräch ist in der ästhetischen Medizin auch deshalb so wichtig, weil sich die Sichtweise auf Altersvorgänge deutlich geändert hat, seit bekannt ist, dass sich Altersvorgänge nicht nur auf die Haut beschränken, sondern alle anatomischen Schichten umfassen. „Patienten sind immer noch der Auffassung, die Hautalterung würde sich nur an der Oberfläche abspielen“, meinte Frau Dr. Patricia Ogilvie, niedergelassene Dermatologin aus München. Insofern ist ihnen auch nicht klar, dass es nicht ausreicht, einzelne Falten aufzufüllen, sondern dass zunächst das durch den Alterungsprozess verlorengegangene

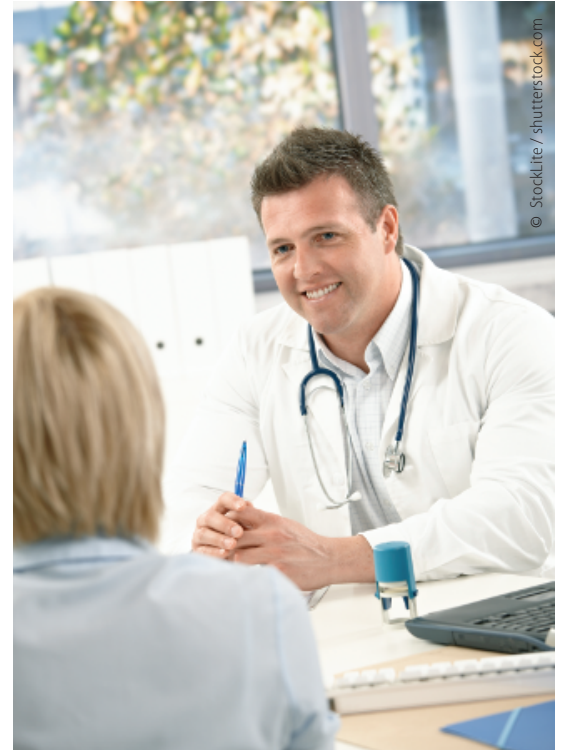

Volumen wieder hergestellt werden muss. Ein Aufklärungsgespräch kann in jedem Fall die Zufriedenheit des Patienten mit dem Behandlungsergebnis verstärken.

(SK)

Pressegespräch „Trends in der ästhetischen Medizin: Warum Qualität zählt" am 24. Mai 2012 in Frankfurt am Main; Veranstalter: Pharm Allergan $\mathrm{GmbH}$, Ettlingen

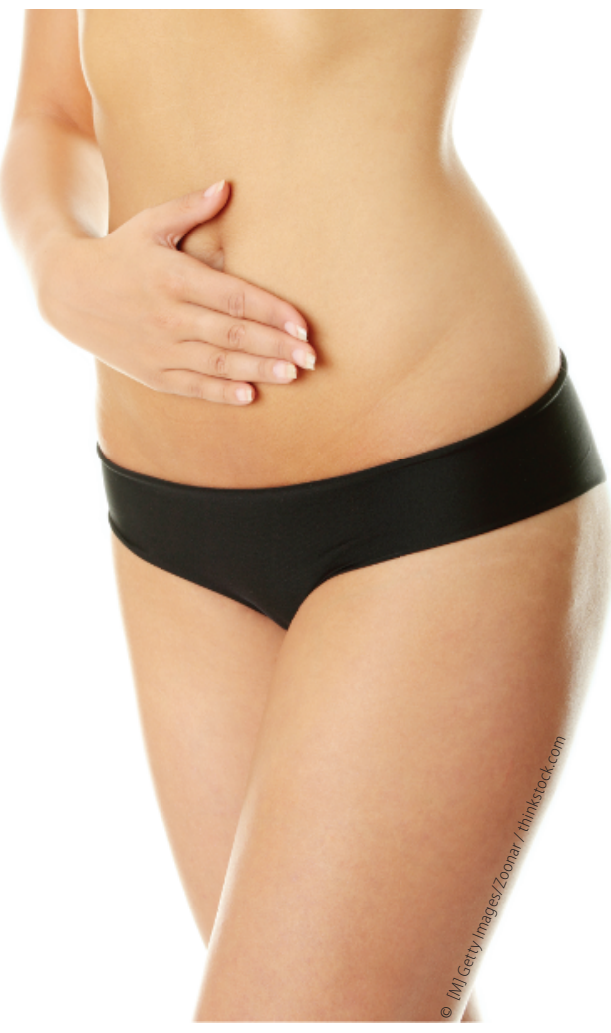

\section{Effektive Hilfe bei Narben und Dehnungsstreifen}

\author{
Auffällige Narben und Dehnungsstreifen können das Selbstbe- \\ wusstsein maßgeblich beeinträchtigen. Viele Menschen mit \\ diesen Makeln fühlen sich dann im wahrsten Sinne des Wortes \\ nicht mehr wohl in ihrer Haut.
}

Das Hautpflegeöl Bi-Oil wurde speziell entwickelt, um das Erscheinungsbild von Narben und Dehnungsstreifen zu verbessern. Die Kombination aus wertvollen Inhaltsstoffen wie Vitamin A, Ringelblumenextrakt, Öl der römischen Kamille, Lavendel- und Rosmarinöl ist mehr als die Summe seiner Teile und pflegt die Haut effektiv. Dank des besonderen Bestandteils PurCellin Oil ist es einerseits so leicht wie eine Lotion, dabei aber gleichzeitig so reichhaltig wie ein Öl. Bi-Oil zieht schnell ein ohne zu fetten und eignet sich für Gesicht und Körper. Wissenschaftliche Wirkungsstudien zeigen innerhalb weniger Wochen eine Verbesserung des optischen Erscheinungsbildes der Haut: Bei Narben zeigte sich eine Verbesserung bei den Probanden nach zwei Wochen um bis zu 65\%. Bei Dehnungsstreifen verbesserte sich das Hautbild der Studienteilnehmer im gleichen Zeitraum um bis zu 90\%. Dies ist das Ergebnis zweier unabhängiger Tests.

Nach Presseinformationen von Delta Pronatura 